\title{
Bioinspired Approach to Silica Nanoparticle Synthesis Using Amine- Containing Block Copoly(vinyl ethers): Realizing Controlled Anisotropy
}

\author{
Ayae Sugawara-Narutaki, ${ }^{* \dagger,}, \nabla$ Sachio Tsuboike, ${ }^{\ddagger}, \nabla$ Yukari Oda, ${ }^{\S, \bigcirc}$ Atsushi Shimojima,,$" \perp$ \\ Kira B. Landenberger, ${ }^{\S} \#$ Tatsuya Okubo,${ }^{\ddagger}$ and Sadahito Aoshima*, \\ 'Department of Materials Chemistry, Nagoya University, Furo-cho, Chikusa-ku, Nagoya 464-8603, Japan \\ *Department of Chemical System Engineering, The University of Tokyo, Hongo, Bunkyo-ku, Tokyo 113-8656, Japan \\ ${ }^{\S}$ Department of Macromolecular Science, Osaka University, Machikaneyama, Toyonaka, Osaka 560-0043, Japan \\ "Department of Applied Chemistry, Waseda University, Okubo-3, Shinjuku-ku, Tokyo 169-8555, Japan \\ ${ }^{\perp}$ Kagami Memorial Research Institute for Materials Science and Technology, Waseda University, Nishiwaseda-2, Shinjuku- \\ ku, Tokyo 169-0051, Japan \\ \#Department of Polymer Chemistry, Kyoto University, Kyoto University Katsura, Nishikyo-ku, Kyoto 615-8510, Japan \\ Author Contributions \\ $\nabla$ A.S-N. and S.T. contributed equally to this article. \\ Present Address \\ ${ }^{\circ}$ Department of Applied Chemistry, Kyushu University, Motooka, Nishi-ku, Fukuoka 819-0385, Japan
}

\section{Table of Contents}

Figure S1. An SEM image of particles formed in the absence of polymers $\quad$ ………...S2

Figure S2. The thermogravimetric and differential thermal analysis (TG-DTA) of samples N1-5 $\quad \cdots \cdots \cdots \cdots \cdots \cdots \cdot . \cdot \mathrm{S} 3$

Figure S3. TEM and SEM images of N1-3 after the removal of the polymers by calcination $\quad$ ……………...S4

Figure S4. SEM images of $\mathbf{N} 4$ and $\mathbf{N} 5$ after the removal of the polymers by calcination

Figure S5. Nitrogen adsorption-desorption isotherms of N1-5 after the removal of the polymers by calcination

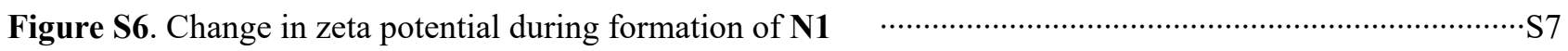

Figure S7. An SEM image of N7 

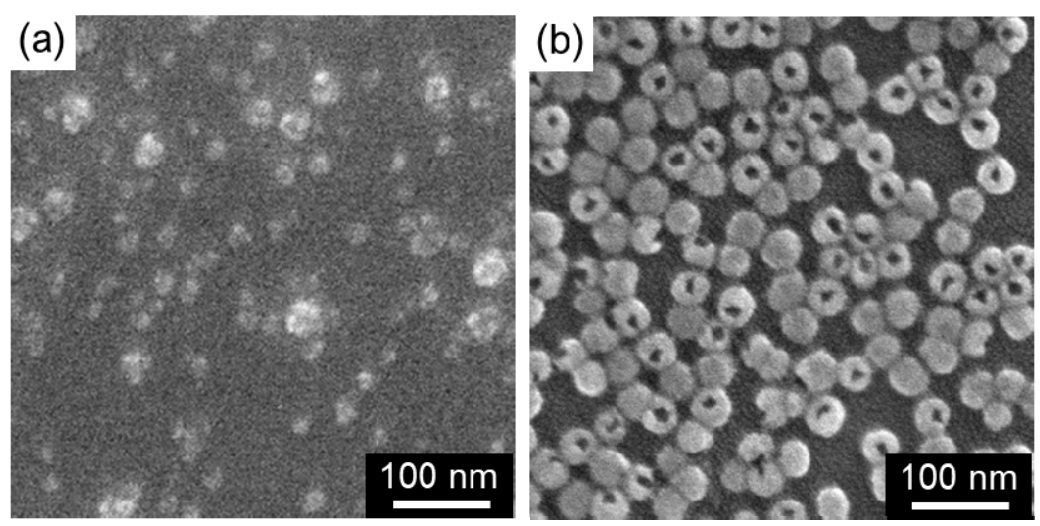

Figure S1. (a) Field-emission scanning electron microscopy (FE-SEM) image of silica nanoparticles formed in the absence of polymers. For comparison, (b) FE-SEM image of N1 (particles formed in the presence of polymer 1). Organic substances or polymers were removed prior to SEM measurements by UV/ozone treatment. 


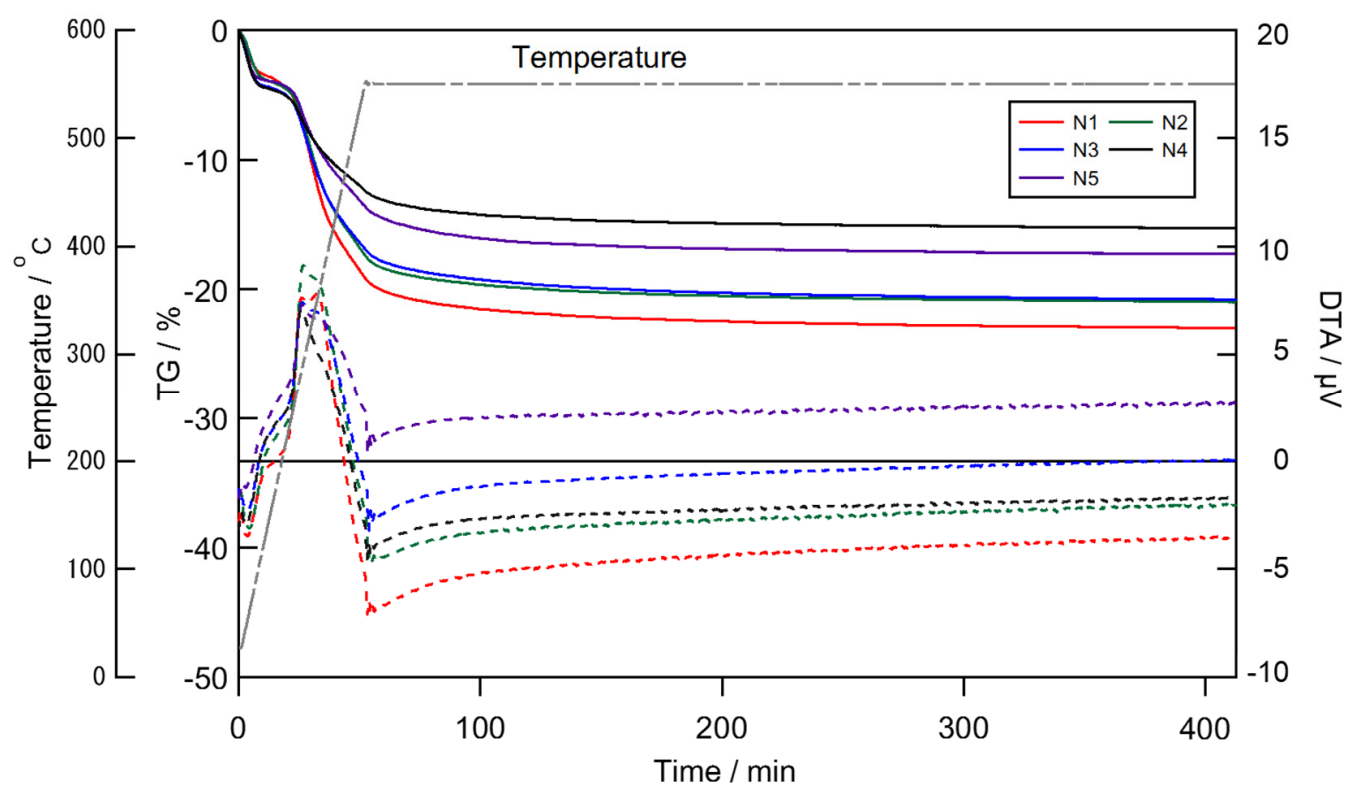

Figure S2. The thermogravimetric (TG) measurements and differential thermal analysis (DTA) of samples N15, measured using a heating rate of $10^{\circ} \mathrm{C} / \mathrm{min}$ under the flow of a 1:9 $\mathrm{O}_{2} / \mathrm{He}$ gas mixture, are shown. The solid lines represent the TG data and the dotted lines show the DTA data. 

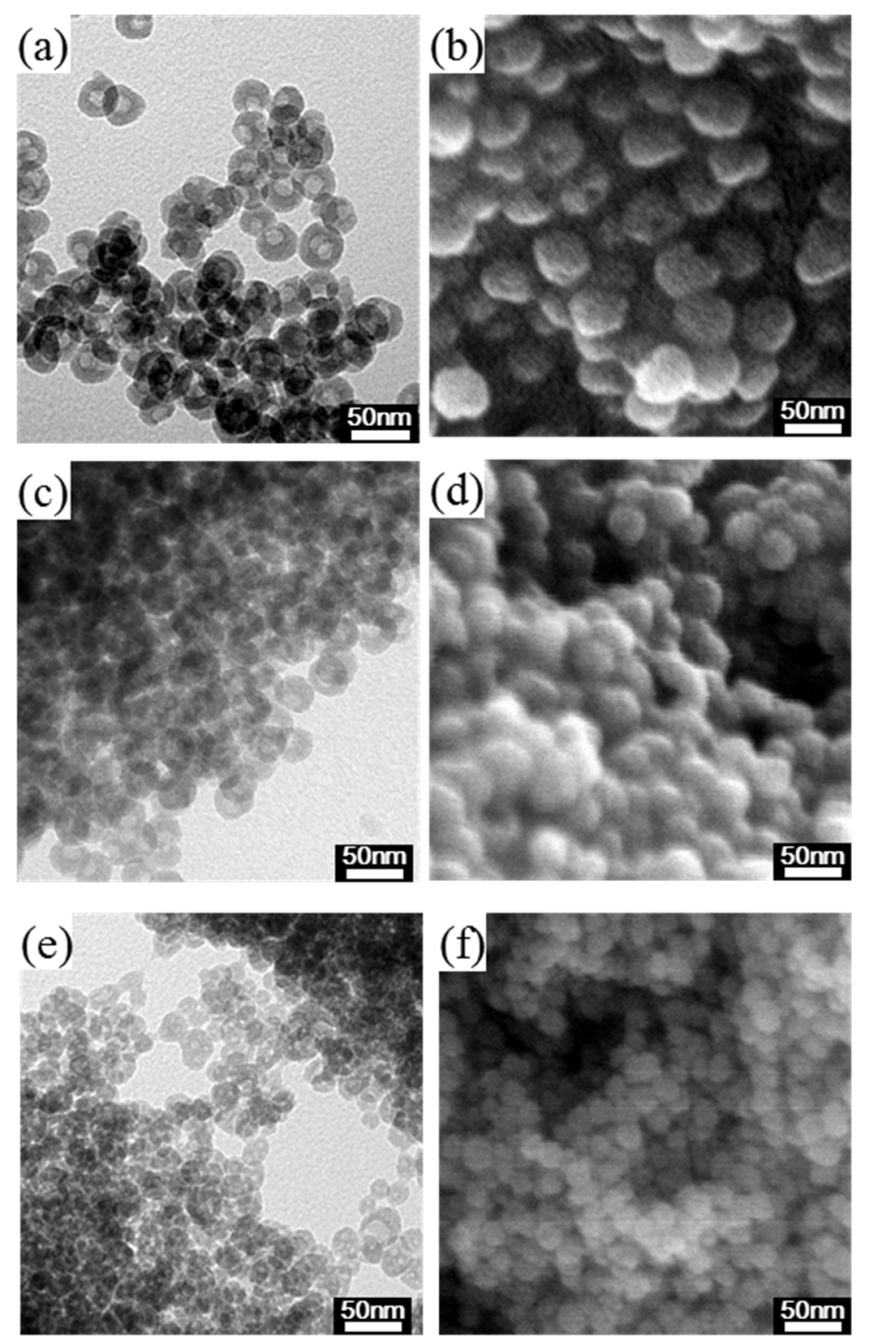

Figure S3. TEM (a,c,e) and SEM (b,d,f) images of N1 $(a, b), \mathbf{N 2}(\mathrm{c}, \mathrm{d})$, and $\mathbf{N 3}(\mathrm{e}, \mathrm{f})$ after the removal of the polymers by calcination. 


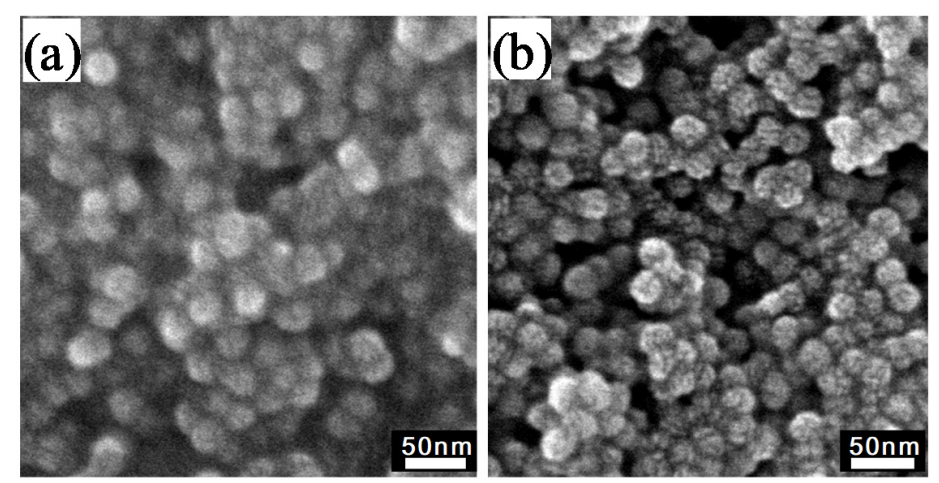

Figure S4. SEM images of N4 (a) and N5 (b) after the removal of the polymers by calcination. 


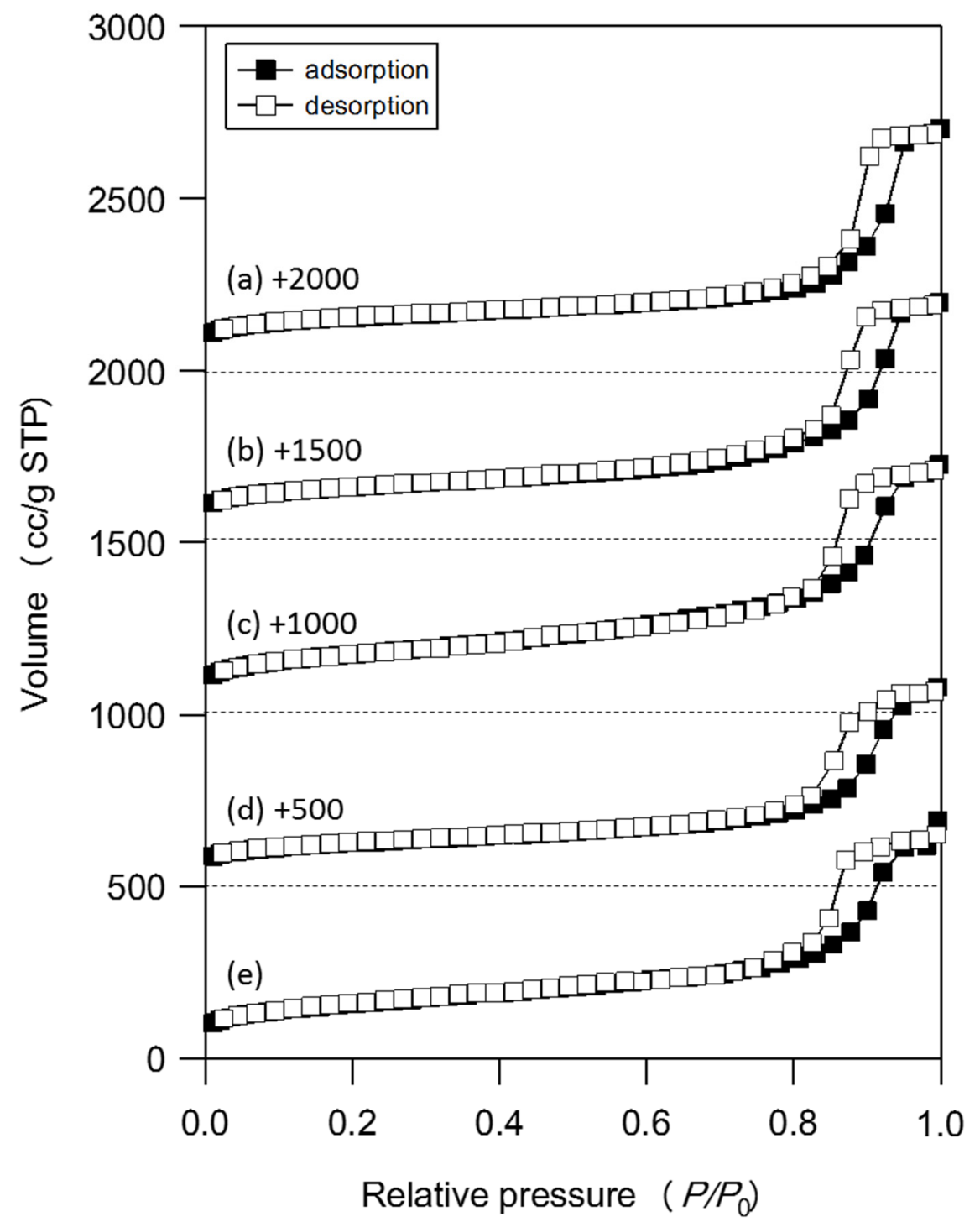

Figure S5. Nitrogen adsorption-desorption isotherms of N1 (a), N2 (b), N3 (c), N4 (d), and N5 (e) after the removal of the polymers by calcination. Adsorption and desorption points are marked by solid ( $\square$ ) and empty ( $\square$ ) squares, respectively. 


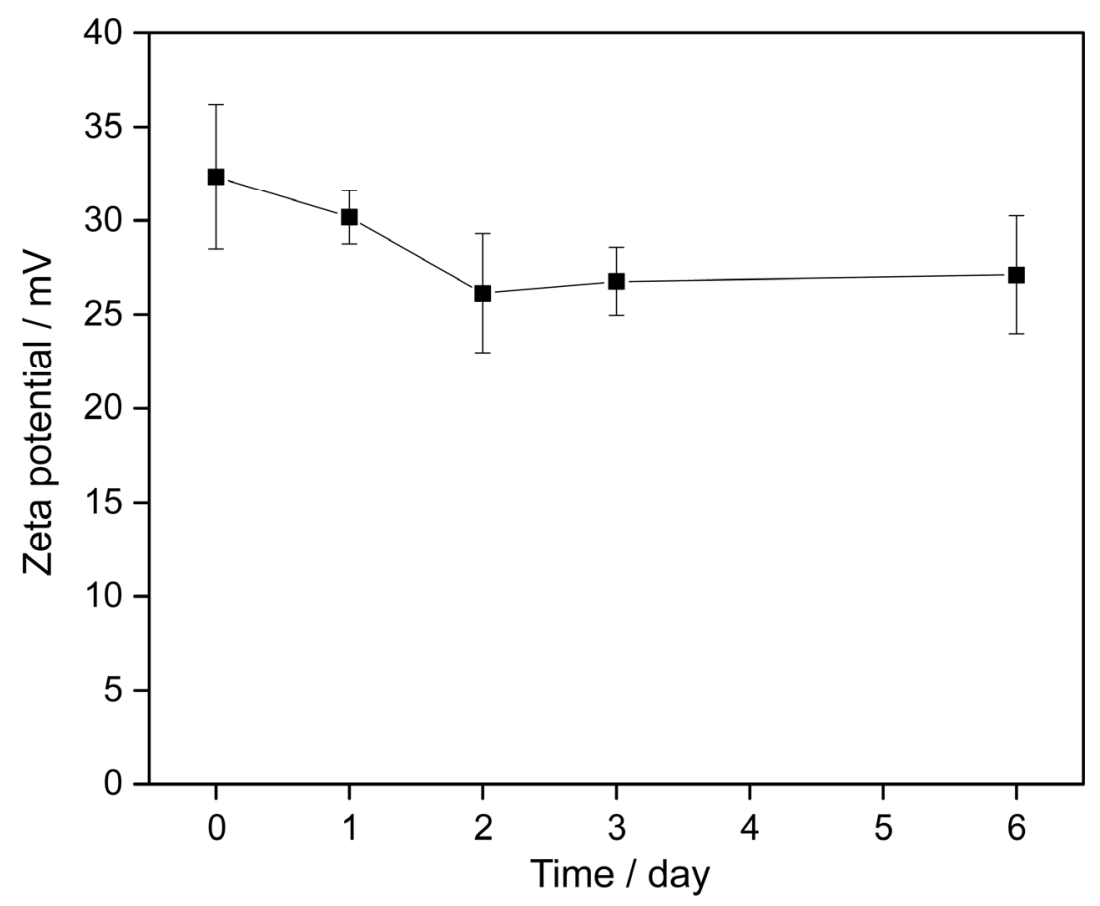

Figure S6. Change in the zeta potential during the formation N1. The value for day 0 corresponds to that of the micelles of polymer $\mathbf{1}$. The plots represent the mean $\pm \mathrm{SD}$. Lines are provided to guide the eye. 


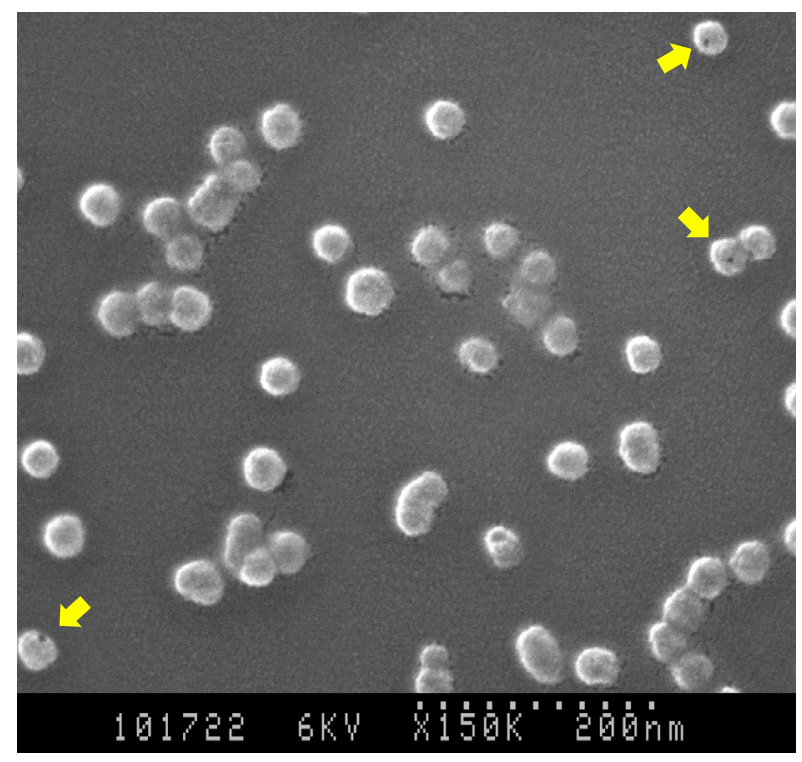

Figure S7. An SEM image of $\mathbf{N} 7$ formed from TEOS $\left(0.04 \mathrm{wt} \%\right.$ as $\left.\mathrm{SiO}_{2}\right)$ and polymer $7(0.01 \mathrm{wt} \%)$. Yellow arrows indicate the particles with openings that are visible in this image. 